\title{
Micromechanics and Macromechanics of the Tensile Deformation of Nacre
}

\author{
H.J. Qi ${ }^{1}$, B.J.F. Bruet ${ }^{3}$, J. S. Palmer ${ }^{2}$, C. Ortiz ${ }^{3}$, M.C. Boyce ${ }^{2}$ \\ ${ }^{1}$ Department of Mechanical engineering, University of Colorado, Boulder, CO, \\ USA qihecolorado.edu \\ ${ }^{2}$ Department of Mechanical Engineering, Massachusetts Institute of Technology, \\ Cambridge, MA, USA mcboyce@mit.edu \\ ${ }^{3}$ Department of Material Science and Engineering, Massachusetts Institute of \\ Technology, Cambridge, MA, USA cortiz@mit.edu
}

\begin{abstract}
Many natural materials exhibit extraordinary combinations of mechanical properties which are achieved through highly tailored and organized hierarchical microstructures. In particular, materials which function as natural body armor, such as mollusk shells, possess a structure with important features and properties at a variety of length scales, from the various constituent building blocks to the overall integrated and synergistic mechanical behavior of their complex assemblies. In this study, the mechanical behavior of the inner "brickand-mortar" nacreous layer of mollusk shells was modeled by taking into account both the mechanical behavior of organic matrix and the geometrical arrangement of the mineral-rich tablets. The protein, Lustrin A, which is present in Haliotis rufescens (red abalone) nacre, has been shown to possess a modular structure consisting of $\sim 10$ domains linked in series. Axial force-extension experiments on the full organic matrix of this same species exhibits an irregular "saw-tooth" type profile, whereupon numerous load drops are found to occur over the course of large axial extension (Smith, et al., 1999). This nanomechanical behavior has been attributed to the sequential unfolding of Lustrin $A$ subunits and their corresponding rupture of sacrificial bonds. The micromechanical model developed here incorporates a new finite deformation constitutive law that assumes sequential force-induced unfolding of the individual protein domains in the organic matrix, as well as the complex spatial organization of the organic and inorganic components. Numerical simulations of tensile extension of representative volume elements of nacre show that progressive unfolding of the modules in the organic matrix provide a macroscopic "softening" mechanism, thus mitigating load transfer to the aragonite tablets, as well as averting early failure of the adhesive layers. This softening mechanism also enables larger deformation of nacre without catastrophic failure, and therefore offers an effective avenue for energy dissipation.
\end{abstract}




\section{Introduction}

Over millions of years of evolution, mollusks have developed an exemplary biological body armor that exhibits a multiscale, hierarchical composite structure. Natural armor ideally must protect against penetration and crash impacts, in particular by absorbing and dissipating energy. Many mollusk shells employ a multi-layered system including a hard outer layer of prismatic calcite crystals as a first barrier to penetration impacts, and an inner layer of nacre which absorbs and dissipates energy through inelastic deformation and fracture processes (Fig. 1).
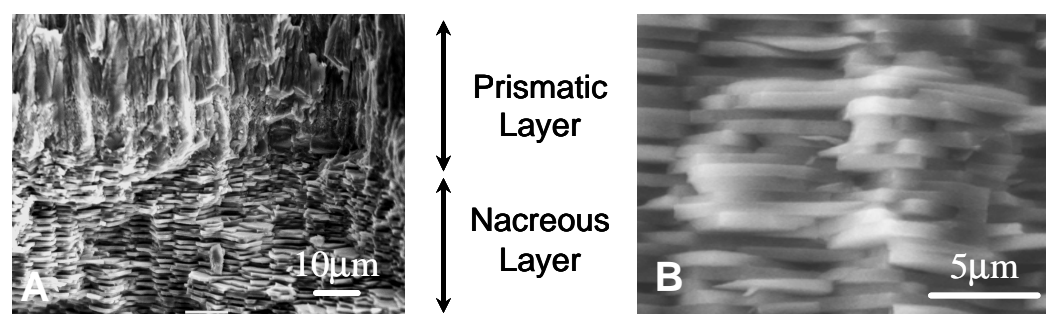

Fig. 1. SEM images of (A) the prismatic - nacreous boundary;

(B) nacreous layer.

Nacre is a biogenic composite consisting of alternating layers of mineral tablets separated by thin layers of a biomacromolecular matrix. Nacre is composed of $\sim 95 \mathrm{wt}$. $\%$ aragonite (i.e. an orthorhombic phase of $\mathrm{CaCO}_{3}$ ) in the form of polygonal tablets which have dimensions of $\sim 5-20 \mu \mathrm{m}$ (perpendicular to the aragonite unit cell $c$-axis) and $\sim 0.5-1.0 \mu \mathrm{m}$ in thickness (parallel to the aragonite unit cell $c$-axis). The remaining $\sim 5 \mathrm{wt}$. $\%$ of nacre is composed of a protein-rich organic matrix $\sim 30-50 \mathrm{~nm}$ in thickness which acts as adhesive layers connecting aragonite tablets, thus maintaining the structural integrity of the composite. Additionally, small ( $30 \mathrm{~nm}$ in diameter) mineral bridges span the tablet layers. Pure aragonite is a very brittle material exhibiting linear elastic behavior prior to brittle failure and is rarely used as an engineering material. However, nacre, through its "brick and mortar" composite structure of micronscale tablets and sub-micron scale adhesive protein-rich layers, enables outstanding mechanical performance including an excellent combination of stiffness, strength, impact resistance, and toughness (Curry, 1977; Weiner and Traub, 1984; Jackson et al., 1988). The ability of nacre to optimize mechanical performance by properly organizing materials at different length scales has attracted a large interest in developing biomimetic materials (e.g., He et al., 1997; Mayer and Sarikaya, 2002), which, in turn, necessitates a deep understanding of the biological mechanisms responsible for its superior properties.

Hence, there has been much discussion recently of the deformation mechanisms of nacre in the literature. As emulated by modern advanced 
composites, the small length scale of the individual aragonite tablets is expected to reduce its defect content (i.e., the Griffith flaw argument), thus providing enhanced strength when compared to that of macroscale monolithic aragonite. This well-known and technologically exploited phenomenon was quantified for the case of the aragonite tablets in nacre using fracture mechanics analysis (Gao, et al. 2004). Jackson, et al. (1988) previously noted that the observed tablet pullout rather than tablet fracture under nacre tensile loading conditions suggested a high tensile strength of the micron-scale mineral tablets. The protein-rich adhesive layers facilitate the overall deformation processes by transferring load to the tablets. Evans et al. (2001) and Wang et al. (2001) studied inelastic deformation events in nacre which were attributed to the formation of dilatation bands and the interactions of nanoscale asperities during pull-out of the mineral tablets. In contrast, Katti et al. (2001, 2002) investigated the elastic and inelastic response of nacre by using a three-dimensional finite element model where the organic matrix was modeled as an elastoplastic material and suggested that the contact between neighboring mineral layers most likely has little effect on the overall elastic properties of nacre. Ji and Gao (2004b) used the virtual internal bond (VIB) model for the protein matrix and found that the macromolecular layer could effectively enhance the toughness of nacre through crack shielding and impact protection, consistent with the experiments of Jackson, et al. (1988).

Recently, nanoscale imaging and mechanical testing techniques have been used to study nacre, revealing new insights on the structure and properties of both the aragonite tablets and the organic matrix. Barthelat and Espinosa (2003) measured the nanoindentation properties of individual nacre tablets from Haliotis rufescens and through a comparison with finite element models suggested that the presence of intertablet organic material up to 3 layers below the tablet tested did not cause significant deviation on the elastic property of aragonite tablet measured. Bruet, et al. (2005) conducted nanoindentation on individual aragonite tablets from the gastropod mollusk Trochus Niloticus and estimated a Young's modulus of $\sim 90 \mathrm{GPa}$ and a yield stress of $\sim 10 \mathrm{GPa}$ through a comparison with an elastic-perfectly plastic finite element model. AFM inspection of the indented region showed extensive pileup around the indentation zone and thus plastic deformation within the tablet; no micro/nanocracks were observed (Fig. 2.). This suggested that the small length scale of the tablet provides a material with a reduced defect content and also suggested that occluded biomacromolecules may play a significant role in the deformation. Smith et al. (1999) used atomic force spectroscopy to test the mechanical axial force-extension behavior of organic matrix strands exposed on a fractured nacre surface. The axial force-extension behavior exhibited an irregular "saw-tooth" character, so named because of the repeating pattern of a nonlinear force increase with extension followed by abrupt load drops. This type of nanomechanical profile is similar to that observed in numerous single molecule tests on other protein molecules (including titin (Rief et al., 1997), tenascin (Oberhauser et al., 1998), and spectrin (Rief et al., 1999; Law, et al., 2003)) and is attributed to unfolding of compacted domains along the 
biomacromolecular chain; this behavior is speculated to play a significant role in the mechanical behavior of nacre.
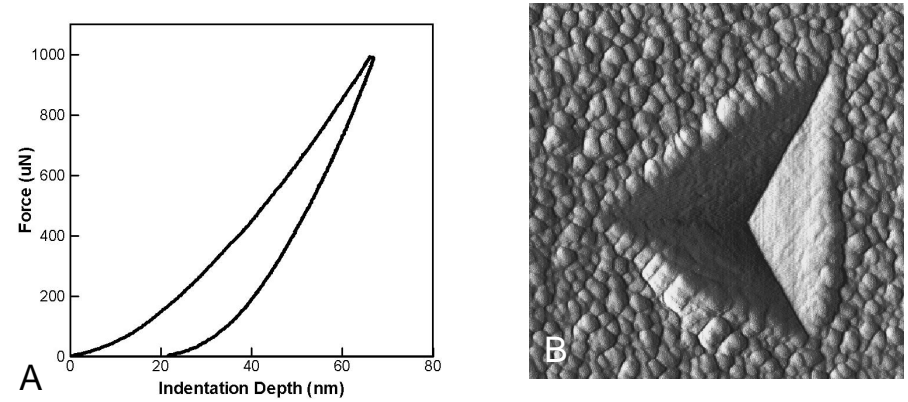

Fig. 2. Nanoindentation of an individual aragonite tablet. (A) averaged force vs indentation depth on individual freshly cleaved T. Niloticus nacre tablets; (B) tapping mode atomic force microscopy image of top down view of residual nanoindentation impression on individual freshly cleaved $T$. Niloticus nacre tablet; The horizontal dimension of B is 3.5 microns. (Bruet et al., 2005).
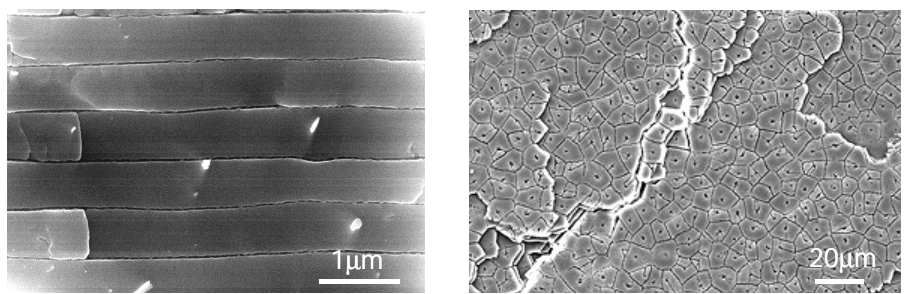

Fig. 3. Scanning electron microscopy (SEM) images of the nanoscale structure of nacre of gastropod mollusk Trochus Niloticus: side view (left) and top view (right) (Bruet et al., 2005).

In this paper, the tensile deformation of nacre is simulated and studied utilizing micromechanical modeling which accounts for the basic features of the brick-and-mortar microstructure as described above. In order to understand the role of the organic matrix in contributing to the overall tensile deformation of nacre, a newly developed finite deformation constitutive model (Qi, et al., 2004) accounting for the influence of unfolding on the stress-strain behavior of the organic matrix is used to study the manner by which the adhesive layers mitigate load transfer to the aragonite tablets. The recent nanoindentation characterization of the tablets (Bruet, et al., 2005) is utilized to properly describe the mechanical properties of the tablets. The micromechanics and the macromechanics of the 
tensile deformation of nacre from the initial linear region into the nonlinear region of the macroscopic stress-strain behavior will thus be simulated.

\section{Micromechanical Model Description}

The micromechanical model has been formulated in terms of two distinct ingredients: (1) an idealized, but adequate, representation of the geometry of the microstructure together with appropriate periodic boundary conditions; and (2) mathematical descriptions of the stress-strain behavior of the constituent materials.

\subsection{Geometrical Representation of the Microstructure}

The microstructure of nacre is one of a three-dimensional arrangement of parallel layers of somewhat randomly staggered polygonal aragonite tablets (planar dimension of $\sim 5 \mu \mathrm{m}$ and thickness of $\sim 1 \mu \mathrm{m}$ ) connected by thin adhesive layers of protein rich matrix (thickness of $\sim 40 \mathrm{~nm}$, Fig. 1B and Fig. 3), noting that small mineral bridges (diameter of $\sim 30 \mathrm{~nm}$ ) are also observed linking parallel layers. Here, in this early stage micromechanical representation, the structure is idealized as two-dimensional (accounting for key geometrical features through the thickness of the nacre). The representative volume element (RVE) considers a periodic staggered arrangement of tablets connected to one another by thin adhesive layers as shown in Fig. 4. Periodic boundary conditions are utilized on all edges and thus only the region indicated by the dashed lines in Fig. 4A (enclosing the RVE) is numerically simulated. The model microstructure dimensions were taken from the SEM micrographs of Bruet, et al. (2005) as: $L=5.8 \mu \mathrm{m}, h_{1}=870 \mathrm{~nm}, t_{1}=t_{3}=40 \mathrm{~nm}$. Other SEM images (e.g. Jackson, et al., 1988) reveal that the organic matrix forms ligands bridging two neighboring aragonite tablets during deformation. To account for this feature, small gaps of width of $h_{2}=1 \mathrm{~nm}$ were introduced in the adhesive layer prior to the application of deformation. We thus are assuming that the constraint imposed by the tablets during extension creates high hydrostatic tension in those adhesive layers normal to the tensile axis (the 1-direction) and some amount of cavitation and ligand formation occurs early on in the deformation. The distance between two neighboring gaps is $h_{3}$ and $h_{3}>h_{2}$. The gap dimensions were deduced from the Jackson, et al. (1988) SEM images of ligands.

Periodic boundary conditions are applied to ensure plane strain tension where the tensile loading axis is the 1-direction, the constraint direction is the 3direction, and the free direction is the 2-direction. Note that the two-dimensional nature of this model means that, although a staggered arrangement of tablets is modeled in the 1-2 plane, the tablets are essentially columns extending in the 3direction and subjected to a constraint of no deformation in the 3-direction. Details on appropriately applying the periodic boundary conditions and then obtaining the macroscopic tensile stress-strain behavior of the RVE are provided in, for example, Danielsson et al. (2002) and Sheng et al. (2004). We note that the 
two-dimensional and periodic nature of this model loses some of the deformation paths available in a fully three-dimensional model of the staggered tablet arrangement, but can still act to identify basic contributions of different features of the microstructure to the overall macroscopic behavior of the composite. Ongoing research addresses more detailed description of the three-dimensional and random multitablet nature of the tablet/mortar structure.
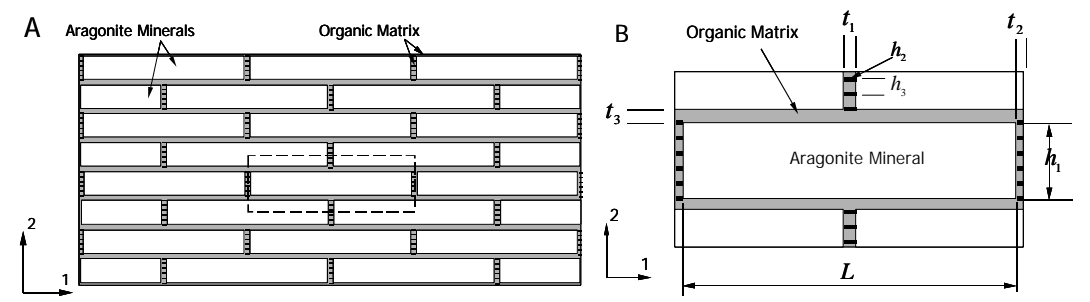

Fig. 4. A schematic of the micromechanical model for nacre. (A) The brick-and-mortar structure; (B) the representative volume element (RVE); Note that the RVE represents a repeating unit (enclosed by dashed line) in (A). The 1-axis is the tensile direction.

\subsection{Constitutive Models of the Stress-Strain Behavior of the Constituent Materials}

Aragonite Tablets: The aragonite tablets and their mineral bridges were modeled as an isotropic elastic-perfectly plastic material. Using the modulus and yield strength estimated from the nanoindentation tests of Bruet et al. (2005) on individual nacre tablets of Trochus Niloticus, the material is taken to have a modulus of $90 \mathrm{GPa}$ and a yield stress of $10 \mathrm{GPa}$. We note that this seemingly high yield stress is consistent with the small size scale of the material volume under investigation. We further note that the size scale and the crystal structure of the aragonite tablets dictates an anisotropic behavior; however, this anisotropy is considered to be a higher order effect within the behavior under investigation in this paper and is being addressed in ongoing research.

Organic Adhesive Layers: The stress-strain behavior of the organic matrix is modeled using a newly developed constitutive model that accounts for the unfolding behavior of compacted domains along the macromolecular chains in the protein network (Qi, et al., 2004). This model is briefly described below.

Using statistical mechanics, the extensional force extension behavior of a macromolecular chain can be represented by a freely-jointed-chain (FJC) model (Kuhn and Grun, 1942; Treloar, 1958), or a worm-like-chain (WLC) model (Kratky and Porod, 1949; Fixman and Kovac, 1973; Marko and Siggia, 1995). 
The FJC model is based on a statistical survey of possible configurations, thus entropy, of a macromolecular chain during stretching. The FJC model relates the axial force $f$ to the chain end-to-end distance $r$ of the macromolecular chain by:

$$
f=\frac{k_{B} T}{l} \beta,
$$

where $k_{B}$ is Boltzmann's constant, $T$ is absolute temperature, $l$ is the Kuhn segment statistical length. $\beta=L^{-1}(r / L)$ is the inverse Langevin function, where $L(\beta)=\operatorname{coth} \beta-1 / \beta$ defines the Langevin function, and $L=N l$ is the contour length of the macromolecular chain where $N$ is the number of "rigid links" along the chain.

To predict unfolding of domains within an individual macromolecular chain during extension, a new criterion is employed whereby the evolution in orientation of rigid links relative to the chain stretch direction is monitored. The rigid links increasingly align towards the stretching direction during extension, thus continually varying their orientation $(\theta)$ distribution, where $\theta$ is defined as the azimuthal angle between each rigid link and the vector connecting the two chain ends. As the chain end-to-end displacement increases, the peak orientation angle, $\theta_{p}$, decreases. Upon reaching a critical value of $\theta_{p}^{c r}$, unfolding of a domain is triggered. The value of $\theta_{p}^{c r}$ is governed by the particular chemistry of bonding within the fold and is to be taken as a material parameter.

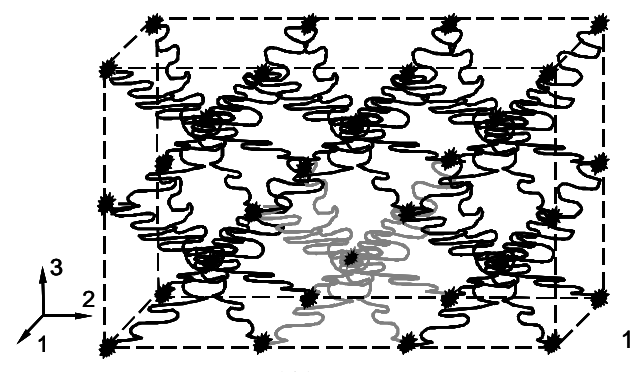

(A)

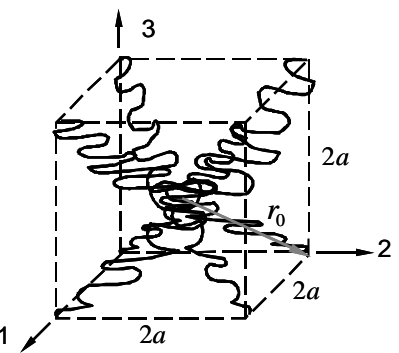

(B)

Fig. 5. Schematics of three-dimensional planar network: (A) An idealized macromolecule network with perfectly staggered crosslinks, before deformation; (B) A 8-chain network, which is the representative volume element (RVE) highlighted in gray in (A).

The single polymer chain behavior is then incorporated into a model for a three-dimensional (3D) macromolecular network solid. Here, a random 3D network is modeled utilizing an idealized "eight-chain" network representation (Fig. 5) (Arruda and Boyce 1993); this network idealization captures the essential features of a random network in an "average" sense. The edges are taken to be 
aligned with the principal stretch directions. The strain energy density calculated based on this RVE subjected to stretch is:

$$
\bar{U}=v k_{B} T N\left[\frac{\lambda_{c}}{\sqrt{N}} \beta_{c}+\ln \left(\frac{\beta_{c}}{\sinh \beta_{c}}\right)-c\right],
$$

where $v$ is density of chains between crosslink sites, $\beta_{c}=\mathrm{L}^{-1}(r / L)=\mathrm{L}^{-1}\left(\lambda_{c} / \sqrt{N}\right)$, $\lambda_{c}=\left(\left(\lambda_{1}^{2}+\lambda_{2}^{2}+\lambda_{3}^{2}\right) / 3\right)^{1 / 2}$ is the chain stretch, and $c$ is the contribution from the initial entropy of the macromolecule. Note that as molecular chain stretch evolves with the macroscopic stretch of the solid, the domains of the macromolecular chains will successively unfold as they reach the critical conditions. The unfolding of the domains acts to increase the contour length of the molecule (i.e. $L$ evolves with stretch conditions) which in turn increases the entropy of the network creating a drop in the stress-strain curve. The network becomes less oriented as a result and the orientation evolution process begins again and the repeating "sawtooth" pattern is thus captured.

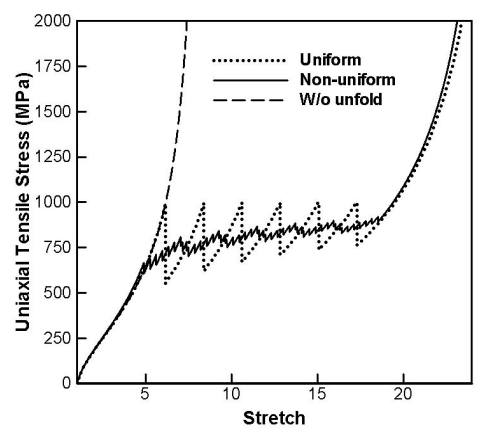

Fig. 6. Finite deformation nominal stress versus stretch behavior of simple tension of a modular three-dimensional network representing the organic matrix in nacre with and without consideration of domain unfolding.

As an illustration, Fig. 6 shows the nominal stress versus stretch curve for simple tension on a three-dimensional solid composed of a network of modular macromolecules (i.e. molecules containing folded domains). The dashed line plot in Fig. 6 shows a simulation where unfolding does not occur during stretching. The dotted line plot in Fig. 6 shows a simulation for a homogeneous macromolecular network where all polymer chains between crosslinks have identical initial contour lengths and hence, all chains reach unfolding conditions at the same macroscopic stretch states. The solid line plot of Fig. 6 is a simulation which considers a nonuniform distribution in initial contour lengths where different polymer chains achieve unfolding conditions under slightly different stretch states. The material properties used for these simulations are listed in 
Table 1.

It is noted that in this case of simple tension, when the chain contour lengths are statistically distributed (solid line, non-uniform in Fig. 6), the stressstrain behavior undergoes a nonlinear increase, then transitions to a plateau region, and finally increases again at large stretches. The first transition from a nonlinear increase to the plateau region corresponds to successive triggering of the unfolding of modules belonging to various polymer chains between crosslinks within the solid. The second transition from the plateau region to a second nonlinear increase corresponds to when all modules have unfolded and increased strain hardening ensues. These two transitions distinguish modular networks as having a unique "softening" behavior, which has also been observed in rubbers and thermoplastic elastomers (Qi and Boyce, 2004a and 2004b). The effect of this behavior on controlling load transfer to the aragonite tablets during nacre deformation will be illustrated and discussed in the results section.

Table 1. Material properties used for the simulations of the organic matrix

\begin{tabular}{lc}
\hline \multicolumn{1}{c}{ Material Properties } \\
\hline Persistence length, $l(\mathrm{~nm})$ & 0.4 \\
Initial contour length $(\mathrm{nm}), L_{i}$ & 10.0 \\
$\begin{array}{l}\text { Increase in contour length due to } \\
\text { unfolding, } \Delta L(\mathrm{~nm})\end{array}$ & 4.0 \\
$\begin{array}{l}\text { Critical peak angle, } \theta_{p} \text { (degree) } \\
\text { Density of polymer chains between }\end{array}$ & 30.0 \\
crosslinks, $v\left(\mathrm{~m}^{-3}\right)$ & $2.43 \times 10^{28}$ \\
\hline
\end{tabular}

\section{Results and Discussion}

Simulation results for the micromechanical model of nacre subjected to tensile loading are presented in terms of the macroscopic stress-strain behavior of the composite and contours of relevant field variables on deformed configurations of the simulated microstructure at different levels of macroscopic strain.

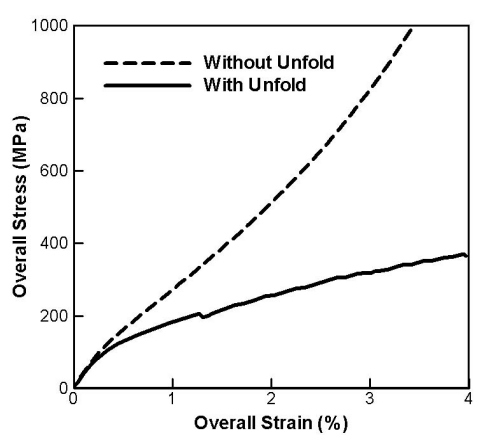


Fig. 7. Macroscopic tensile stress-strain curve for the micromechanical simulations of nacre considering an organic matrix layer with unfolding and one without unfolding.

Fig. 7 depicts the macroscopic tensile stress-strain behavior of the nacre micromechanical model. Two results are shown, one where the organic matrix is modeled neglecting the unfolding of the compacted domains and one where the effects of unfolding are included (the simple tensile stress-strain behavior of these two matrix representations were shown in Fig. 6). The stress-strain results predict an initial modulus of $40 \mathrm{GPa}$, compared to the experimentally reported value of 60 GPa (Jackson, et al., 1988). Note that the Jackson, et al (1988) experiments were on Pinctada nacre as opposed to the Trochus Niloticus nacre from which we took the microstructural parameters; therefore, this comparison should not be perfect, but is given to demonstrate that the simulation results are quantitatively realistic. When unfolding is included in the organic matrix layers, the macroscopic stressstrain curve exhibits an apparent "yield" stress of $135 \mathrm{MPa}$, corresponding to the Jackson, et al (1988) experimental tensile yield strength of $140 \mathrm{MPa}$ which corresponded to tablet pullout as the tensile failure mechanism. The "yield stress" and nonlinear transition corresponds to unfolding events and softening in the organic layers. These unfolding events, in turn, mitigate load transfer to the aragonite tablets, thus preventing the stress levels in the aragonite from reaching fracture level conditions. The post-yield macroscopic strain hardening regime is due to the deformation response of the organic matrix layers parallel to the tensile direction, which shear and transfer load to the tablets via the well-known shear lag load transfer mechanism in fiber and platelet reinforced composites.

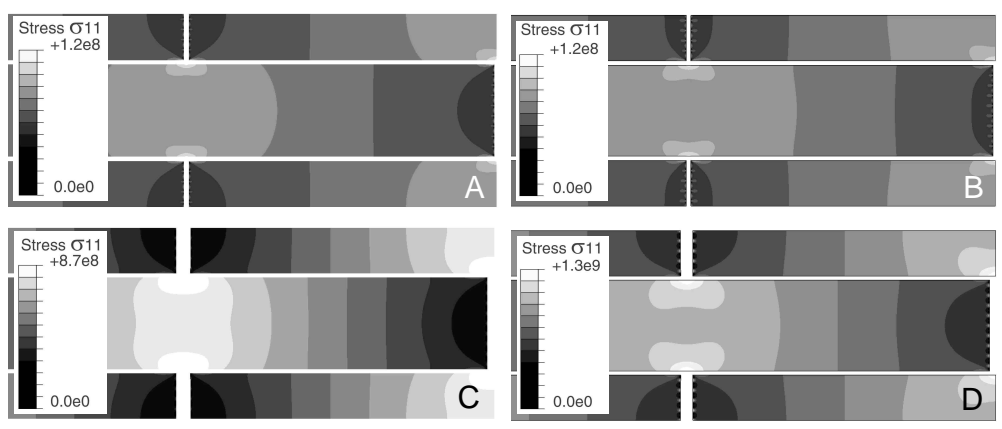

Fig. 8. Contours of the $\mathrm{S}_{11}$ (in $\mathrm{Pa}$ ) in the aragonite tablets for (A) the case of unfolding in organic matrix at $0.2 \%$ overall strain; (B) the case without unfolding at $0.2 \%$ overall strain; (C) the case of unfolding in organic matrix at $2.0 \%$ overall strain; (D) the case without unfolding at $2.0 \%$ overall strain. The 1direction (the loading direction) is the horizontal direction. Note that, due to the dramatically different magnitudes in stress, 
different contour scales are needed and used in A, B, C, and D.

Fig. 8 shows contours of the axial stress $\sigma_{11}$ (in Pascals) in the aragonite tablets at the macroscopic axial strain of $0.2 \%$ (Fig. 8A) and $2.0 \%$ (Fig. 8C) for the case where unfolding of the organic matrix is taken into account. The corresponding stress contours for the case of a matrix without unfolding are shown in Fig. 8B and 8D, respectively. At small tensile strain $(0.2 \%)$ the simulated stress-strain curves for nacre with and without unfolding of the macromolecular component are approximately the same (Fig. 7). Hence, the stress contours in Fig. 8A and 8B appear similar as well. For both the folding and unfolding cases, the tensile stress in the aragonite tablets increases along the tablet length from a value of $70 \mathrm{MPa}$ at the tablet edges (edges normal to the loading direction) to a maximum tensile stress of $\sim 120 \mathrm{MPa}$. This stress buildup results from shearing of the organic adhesive layers between tablets acting to transfer additional load to the tablets as shown in the tablet shear stress contours of Fig. 9.

At a larger overall tensile strain of $\sim 2.0 \%$, which is in the "post-yield" region of the stress-strain curve of Fig. 7 that includes unfolding, the aragonite axial stress builds from a value of about $140 \mathrm{MPa}$ at the tablet edges to a very localized peak value of $870 \mathrm{MPa}$ at the outer edge of the central region of the tablet with an average value of $600 \mathrm{MPa}$ in that region. The buildup of stress from the edge to the center of the tablet is again through shear transfer from the matrix as shown in the corresponding shear stress contours of Fig. 9. In contrast, the axial stress contours at $2.0 \%$ strain when matrix unfolding is not taken into account (Fig. 8D) show a build up from a tablet edge stress of $250 \mathrm{MPa}$ to a central stress (over the entire central section) of over $1.1 \mathrm{GPa}$. This higher tablet stress and its occurrence over a larger volume of the aragonite create a scenario more favorable to tablet fracture. As macroscopic strain continues to increase, these values will continue to rise in a manner consistent with the steep slope of its macroscopic stress-strain curve of Fig 7. In contrast, matrix unfolding substantially reduces the stress levels in the tablets and the manner in which the stress levels increase with increasing macroscopic strain, making tablet pullout the experimentally observed mode of tensile failure instead of tablet fracture.

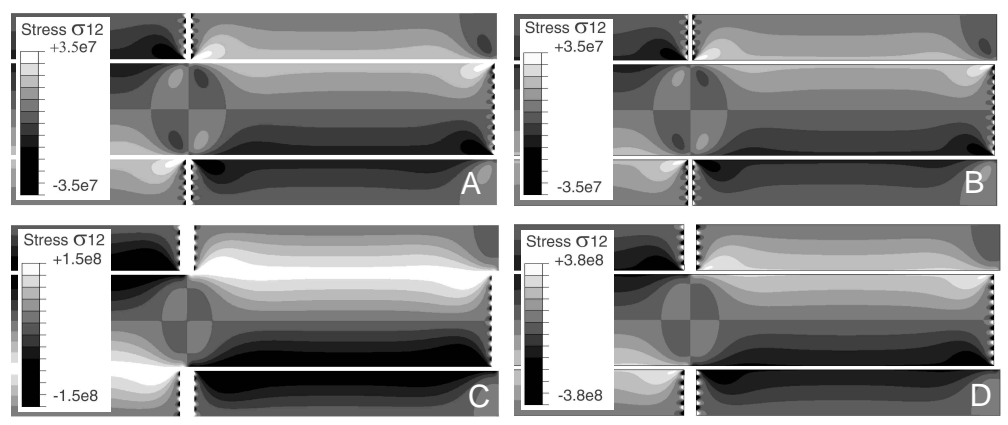


Fig. 9. Contours of $\sigma_{12}$ (in $\mathrm{Pa}$ ) in the aragonite tablets for (A) the case of unfolding in organic matrix at $0.2 \%$ overall strain; (B) the case without unfolding at $0.2 \%$ overall strain; (C) the case of unfolding in organic matrix at $2.0 \%$ overall strain; (D) the case without unfolding at $2.0 \%$ overall strain.

To further illustrate the role of unfolding in controlling load transfer to the tablets during tensile loading conditions, contours of axial stress in the matrix layers normal to the tensile axis at $0.2 \%$ and $2 \%$ strain are shown in Fig. 10. Fig.10A and $10 \mathrm{C}$ show the axial stress in the matrix ligaments at $0.2 \%$ and $2.0 \%$ macroscopic strain when matrix unfolding is not considered; Figures $10 \mathrm{~B}$ and $10 \mathrm{D}$ show the corresponding cases when unfolding is taken into account. The stress levels at $0.2 \%$ strain are similar for both matrix cases $(\sim 100 \mathrm{MPa}$ peak); however, at $2.0 \%$ macroscopic strain the stress in the unfolding matrix are significantly lower than those in the non-unfolding case since unfolding essentially plateaus the stress level in the matrix ligaments. Thus, the stress levels transmitted to the tablets are mitigated by the unfolding events in the matrix ligaments.

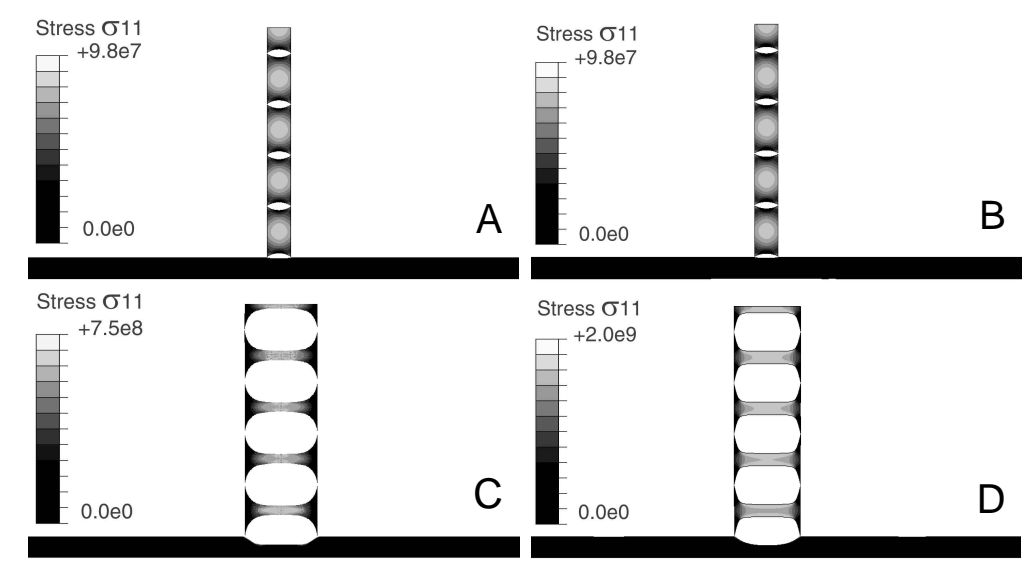

Fig. 10. Contours of stress component $\mathrm{S}_{11}$ (in $\mathrm{Pa}$ ) in the matrix ligaments for (A) the case of unfolding in organic matrix at $0.2 \%$ overall strain; (B) the case without unfolding at $0.2 \%$ overall strain; (C) the case of unfolding in organic matrix at $2.0 \%$ overall strain; (D) the case without unfolding at $2.0 \%$ overall strain. 


\section{Conclusion}

This paper investigated the mechanics of nacre using a micromechanical representative volume element model, where the protein-rich organic matrix is modeled by a recently developed finite deformation constitutive model that accounts for the unfolding of protein molecules in the organic matrix. Numerical simulations of tensile deformation showed that in the presence of unfolding in the organic matrix, the macroscopic stress-strain behavior demonstrated nonlinear behavior with an initial linear elastic region followed by an apparent "yield" stress. This yield was due to triggering unfolding events in the organic layers normal to the applied tension and corresponded to a mitigation of load transfer to the aragonite tablets, thus preventing the stress levels in the aragonite from reaching fracture level conditions. As macroscopic strain was continued to increase, the macroscopic post-yield behavior showed strain hardening. This hardening behavior was due to shear in the organic layers that transferred load to the tablets via the well-known shear lag load transfer mechanism in fiber and platelet reinforced composites. It is noted that this is a preliminary study where the simplified micromechanical model presented here captures the basic structural features of nacre and revealed new understanding of the role of the organic matrix softening mechanisms in controlling load transfer at the microscopic lengthscale. More detailed models which consider the three-dimensional and the random distribution of the tablet and mortar structure are currently under investigation.

Acknowledgments. This research was supported by the U. S. Army through the Institute for Soldier Nanotechnologies, under Contract DAAD-19-02-D0002 with the U.S. Army research office. The content does not necessarily reflect the position of the government and no official endorsement should be inferred. HJQ would also like to acknowledge the support from the start-up fund from the University of Colorado at Boulder.

\section{References}

Arruda, E.M., and M.C. Boyce. 1993. A Three-dimensional constitutive model for the large stretch behavior of elastomers. J. Mech. Phys. Solids 41:389412.

Barthelat, F., and Espinosa, H.D. (2003). Elastic properties of nacre aragonite tablets. Proceedings of the 2003 SEM Annual Conference and Exposition on Experimental and Applied Mechanics, Paper 187.

Barthelat, F., and Espinosa, H.D. (2004). Mechanical Properties of Nacre Constituents: An Inverse Method Approach. MRS fall conference, Boston, MA.

Bruet, B.J.F., Panas, R., Tai, K., Frick, L., Ortiz, C., Qi, H.J, and Boyce, M.C. (2005). Nanoscale morphology and indentation of individual nacre 
tablets from the gastropod mollusk Trochus Niloticus. Submitted for publication consideration, January 2005.

Curry, J.D. (1977). Mechanical properties of mother of pearl in tension. Proc. $R$. Soc. Lond. B. 196:443-463.

Danielsson, M., Parks, D.M., and Boyce, M.C. (2002). Three-dimensional micromechanical modeling of voided polymeric materials. J. Mech. Phys. Solids. 50:351-379.

Evans, A.G., Suo, Z., Wang, R.Z., Aksay, I.A., He, M.Y., and Hutchinson, J.W. (2001). Model for the robust mechanical behavior of nacre. J. Mater. Res. 16:2475-2484.

Fixman, M., and Kovac, J. (1973). Polymer conformational statistics. III Modified Gaussian models of stiff chains. J. Chem. Phys. 58:1564-1568.

Gao, H., Ji, B., , Jager, I.L., Arzt, E., and Fratzl, P. (2003). Materials become insensitive to flaw at nanoscale: Lessons from nature. PNAS, 100:55975600 .

He, J.L., Wang, J., Li, W.Z., and Li, H.D. (1997). Simulation of nacre with TiN/Pt multilayers and a study of their mechanical properties. Mater. Sci. Eng. B49:128-134.

Jackson, A.P., Vincent, J.F.V. and Turner, R.M. (1988). The mechanical design of nacre. Proc. R. Soc. Lond. B. 234:415-440.

Ji, B., and Gao, H. (2004). A study of fracture mechanisms in biological nanocomposites via the virtual internal bond model. Mater. Sci. Eng. A366:96-103.

Katti, D., Katti, K., Tang, J., and Sarikaya, M. (2002). Effect of nanostructure in nacre: a multiscale modeling approach. Proceedings of $15^{\text {th }}$ ASCE Engineering Mechanics Conference, Columbia University, New York, NY.

Katti, D.R., Katti, K.S., Sopp, J.M., and Sarikaya, M. (2001). 3D finite element modeling of mechanical response in nacre-based hybrid nanocomposites. Compu. Theo. Polym. Sci. 11:397-404.

Kratky, O., and Porod, G. (1949). Röntgenuntersuchung gelöster fadenmoleküle. Recl. Trav. Chim. Pays-Bas. 68:1106-1122.

Kuhn, W., and Grun, F. (1942). Beziehungen zwischen elastischen Konstanten und Dehnungsdoppelbrechung hochelastischer Stoffe. Kolliod Z. $101: 248-271$.

Law, R., P. Carl, S. Harper, P. Dalhaimer, D. Speicher, D., and . Discher, D.E. (2003) Cooperativity in forced unfolding of tandem spectrin repeats. Biophys. J., 84:533-544.

Marko, J.F., and Siggia, E.D. (1995). Stretching DNA. Macromolecules 28: 8759-8770.

Mayer, G., and Sarikaya, M. (2002). Rigid biological composite materials: structural examples for biomimetic design. Exp. Mech. 42:395-403.

Oberhauser, A.F., P.E. Marszalek, H.P. Erickson, and J.M. Fernandez. (1998). The 
molecular elasticity of the extracellular matrix protein tenascin. Nature, 393:181-185.

Qi, H.J., Ortiz, C., and Boyce, M.C. (2004). A constitutive model for the stressstrain behavior of biomacromolecular networks containing folded domains. Submitted to for publication , 2004.

Qi, H.J., and M.C. Boyce. (2004a). Constitutive model for stretch-induced softening of the stress-stretch behavior of elastomeric materials. J. Mech. Phys. Solids 52:2187-2205.

Qi, H.J., and M.C. Boyce. (2004b). Stress-strain behavior of thermoplastic polyurethane. Mech. Mater. in press, web-release November, 2004.

Rief, M., M. Gautel, F. Oesterhelt, J.M. Fernandez, and H.E. Gaub. (1997). Reversible unfolding of individual titin immunoglobulin domains by AFM. Science, 276:1109-1112.

Rief, M., J. Pascual, M. Saraste, and H. E. Gaub. (1999). Single molecule force spectroscopy of spectrin repeats: low unfolding forces in helix bundles. $J$. Mol. Biol.,286:553-561.

Smith, B.L., T.E. Schaffer, M. Viani, J.B. Thompson, N.A. Frederick, J. Kindt, A. Belcher, G.D. Stucky, D.E. Morse, and P.K. Hansma. (1999). Molecular mechanistic origin of the toughness of natural adhesives, fibres and composites. Nature, 399:761-763.

Sheng, N., Boyce, M.C., Parks, D.M., Rutledge, G.C, Abes, J.I., and Cohen, R.E. (2004) Multiscale micromechanical modeling of polymer/clay nanocomposites and the effective clay particle. Polymer. 45:487-506.

Treloar, L.R.G. (1958). The Physics of Rubber Elasticity. Oxford at the Clarendon Press.

Wang, R.Z., Suo, Z., Evans, A.G., Yao, N., and Aksay, I.A. (2001). Deformation mechanisms in nacre. J. Mater. Res. 16:2485-2493.

Weiner, S., and Traub, W. (1984). Macromolecules in mullusc shells and their functions in biomineralization. Phil. Trans. R. Soc. Lond. B. 304:425433 